Emily Littlejohn, DO, MPH

Department of Rheumatologic and Immunologic Disease,

Cleveland Clinic

\title{
Keeping lupus patients on hydroxychloroquine during the COVID-19 pandemic
}

\section{Posted May 1, 2020}

\begin{abstract}
Hydroxychloroquine (HCQ) is in short supply as a result of the coronavirus disease 2019 (COVID-19) pandemic, presenting a challenge to rheumatologists to ensure their patients with systemic lupus erythematosus (SLE) continue to take this essential drug. HCQ is the only SLE treatment shown to increase survival and any change in the HCQ regimen is potentially dangerous. Changes in the HCQ regimen should be made jointly with the patient after a discussion of the available evidence and expert opinion and the patient's preferences. Providers need to make thoughtful, informed decisions in this time of medication shortage.
\end{abstract}

\section{LUPUS AND HCQ}

Hydroxychloroquine (HCQ) is in short supply as a result of the coronavirus disease 2019 (COVID-19) pandemic, presenting a challenge to rheumatologists to ensure their patients with systemic lupus erythematosus (SLE) continue to take this essential drug, the only SLE treatment shown to increase survival., ${ }^{1,2}$

Although the optimal daily dose of HCQ in SLE is a subject of controversy, it is typically $5 \mathrm{mg} / \mathrm{kg}$ of real body weight per day in accordance with the 2016 American Academy of Ophthalmology recommendations to avoid ocular adverse effects including corneal deposits, posterior subcapsular cataract, ciliary body dysfunction, and toxic retinopathy. HCQ is available in 200-mg tablets, and many patients take different daily doses to maintain a weekly average of $5 \mathrm{mg} / \mathrm{kg} /$ day: for example, $400 \mathrm{mg}$ one day, and $200 \mathrm{mg}$ the next day.

While there is much to be elucidated regarding

The statements and opinions expressed in COVID-19 Curbside Consults are based on experience and the available literature as of the date posted. While we try to regularly update this content, any offered recommendations cannot be substituted for the clinical judgment of clinicians caring for individual patients.

doi:10.3949/ccjm.87a.ccc023 dosing and monitoring of optimal therapeutic blood levels, recent studies have found that patients with HCQ levels $750 \mathrm{ng} / \mathrm{mL}$ or higher had both a clinically meaningful and statistically significant decrease in disease activity. ${ }^{3}$ Conversely, noncompliance resulting in a low blood HCQ concentration has been shown to be a marker and predictor of SLE exacerbation. ${ }^{4}$ Further, and most relevant to the current COVID-19 shortage dilemma, discontinuation of HCQ in patients with quiescent disease was shown to be associated with a 2.5-fold increase in the risk of new clinical manifestations or, in the case of previous manifestations, a recurrence or an increase in severity. ${ }^{5}$ These studies underscore the importance of uninterrupted therapy in our SLE patients.

In light of the current HCQ shortage related to the COVID-19 pandemic, the American College of Rheumatology's newest guideline on allocating this scarce resource states that, based on shared decisionmaking with the patient, it is reasonable to "pursue HCQ dose reductions and extend HCQ dosing intervals tailored to the individual patient's needs when faced with HCQ shortages." 6 The practices I have instituted within my patient population with these guidelines in mind are as follows:

In patients who are pregnant and in patients whose disease is or has been severe or difficult to control, the risk of flare due to loss of efficacy from subtherapeutic HCQ blood levels is too high, and I would not modify the dosing regimen. Proper coding emphasizing SLE acuity and organ involvement when ordering prescriptions for a 90-day supply and direct discussion with a pharmacist or a peer-to-peer review is often necessary in these situations.

In stable patients with mild disease, it may be more reasonable to conditionally recommend a dosing adjustment. Patients on more than $200 \mathrm{mg} /$ day may decrease to $200 \mathrm{mg}$ daily (1 tablet daily), and those on $200 \mathrm{mg}$ daily may take their dose every other day. 
This strategy is implemented in the hope that blood levels will decrease slowly enough to mitigate fast shifts and subsequent flares.

Any change in the HCQ regimen is potentially dangerous, and any change should be made jointly with the patient after a discussion of the available evidence and expert opinion and the patient's preferences. HCQ is too precious a resource to our SLE patients to take blanket action. As providers, we need to make thoughtful, informed decisions in this time of medication shortage.

\section{REFERENCES}

1. Ruiz-Irastorza G, Egurbide MV, Pijoan Jl, et al. Effect of antimalarials on thrombosis and survival in patients with systemic lupus erythematosus. Lupus 2006; 15(9):577-583. doi:10.1177/0961203306071872

2. Alarcón GS, McGwin G, Bertoli AM, et al; LUMINA Study Group.
Effect of hydroxychloroquine on the survival of patients with systemic lupus erythematosus: data from LUMINA, a multiethnic US cohort (LUMINA L). Ann Rheum Dis 2007; 66(9):1168-1172. doi:10.1136/ard.2006.068676

3. Garg S, Unnithan R, Hansen KE, Costedoat-Chalumeau N, Bartels $\mathrm{CM}$. The clinical significance of monitoring hydroxychloroquine levels in patients with systemic lupus erythematosus: a systematic review and meta-analysis. Arthritis Care Res (Hoboken) 2020; Jan 31. doi:10.1002/acr.24155

4. Costedoat-Chalumeau N, Amoura Z, Hulot JS, et al. Low blood concentration of hydroxychloroquine is a marker for and predictor of disease exacerbations in patients with systemic lupus erythematosus. Arthritis Rheum 2006; 54(10):3284-3290. doi:10.1002/art.22156

5. Canadian Hydroxychloroquine Study Group. A randomized study of the effect of withdrawing hydroxychloroquine sulfate in systemic lupus erythematosus. N Engl J Med 1991; 324(3):150-154. doi:10.1056/NEJM199101173240303

6. American College of Rheumatology. Guiding principles from the American College of Rheumatology for scarce resource allocation during the COVID-19 pandemic: the case of hydroxychloroquine. https://www.rheumatology.org/Portals/0/Files/Guiding-PrinciplesScarce-Resource-Allocation-During-Covid-19.pdf 